TRANSACTIONS OF THE

AMERICAN MATHEMATICAL SOCIETY

Volume 354, Number 12, Pages 5063-5085

S 0002-9947(02)03041-6

Article electronically published on June 10, 2002

\title{
DISCRETE MORSE THEORY AND THE COHOMOLOGY RING
}

\author{
ROBIN FORMAN
}

\begin{abstract}
In Morse Theory for Cell Complexes, we presented a discrete Morse theory that can be applied to general cell complexes. In particular, we defined the notion of a discrete Morse function, along with its associated set of critical cells. We also constructed a discrete Morse cocomplex, built from the critical cells and the gradient paths between them, which has the same cohomology as the underlying cell complex. In this paper we show how various cohomological operations are induced by maps between Morse cocomplexes. For example, given three discrete Morse functions, we construct a map from the tensor product of the first two Morse cocomplexes to the third Morse cocomplex which induces the cup product on cohomology. All maps are constructed by counting certain configurations of gradient paths. This work is closely related to the corresponding formulas in the smooth category as presented by Betz and Cohen and by Fukaya.
\end{abstract}

\section{INTRODUCTION}

In [5] we introduced a discrete version of Morse theory, which could be applied to very general cell complexes. The reader can also see 4, [7, for introductory surveys, [6], [8] and [15] for extensions of the theory, and [9, [3], 1], [13, 14] and [16] for applications of the theory. In this paper we show how the ring structure of the cohomology of a simplicial complex can be seen from the point of view of discrete Morse theory. More generally, given any map on the cohomology ring which is induced by a cochain map, and any collection of discrete Morse functions, we give an explicit procedure for realizing the map by a map on the appropriate space of Morse cochains. Betz and Cohen [2], and, independently, Fukaya [11], [12, showed how one can see the ring structure of the cohomology of a smooth manifold from the point of view of standard Morse theory. Although we follow a slightly different point of view, the main ideas in this paper find their roots in these references. In particular, the idea of describing the relevant cochain maps in terms of graphs (see Figures $0.1-0.7$ below) first appeared in these papers.

Let us quickly review the basics of discrete Morse theory. The reader should consult $[5$ for a more complete presentation. Let $M$ be a finite simplicial complex. For any simplex $a$ of $M$, we will sometimes write $a^{(p)}$ to indicate that $a$ has dimension $p$. For simplices $a$ and $b$ of $M$, we will write $a<b$ or $b>a$ to indicate that $a$ is a face of $b$. A function

$$
f:\{\text { simplices of } M\} \rightarrow \mathbb{R}
$$

Received by the editors August 13, 2001 and, in revised form, January 30, 2002.

2000 Mathematics Subject Classification. Primary 57Q99; Secondary 58E05.

This work was partially supported by the National Science Foundation. 
is a (discrete) Morse function if for each $p$-simplex $a$

$$
\#\left\{b^{(p+1)}>a \mid f(b) \leq f(a)\right\} \leq 1
$$

and

$$
\#\left\{b^{(p-1)}<a \mid f(b) \geq f(a)\right\} \leq 1 .
$$

We say that $a$ is a critical simplex of $f$ if

$$
\#\left\{b^{(p+1)}>a \mid f(b) \leq f(a)\right\}=0
$$

and

$$
\#\left\{b^{(p-1)}<a \mid f(b) \geq f(a)\right\}=0 .
$$

The main theorem of this Morse theory is the following.

Theorem 0.1. $M$ is homotopy equivalent to a $C W$ complex with exactly one cell of dimension $p$ for each critical simplex of dimension $p$.

It is worthwhile to point out that on every simplicial complex $M$ there is a trivial Morse function $f$ which assigns to each simplex its dimension (that is, $f(a)=$ dimension $(a)$ for every simplex $a$ ). For this Morse function, every $\operatorname{simplex}$ of $M$ is critical.

Let $\mathcal{M}^{p} \subseteq C^{p}(M, \mathbb{Z})$ denote the integer cochains which are supported on the critical simplices of $f$ (we will write $\mathcal{M}^{p}(f)$ when we wish to emphasize the function $f)$. By Theorem 0.1, there is a differential $\tilde{\delta}$, so that

$$
\mathcal{M}^{*}: 0 \longleftarrow \mathcal{M}^{n} \stackrel{\tilde{\delta}}{\longleftarrow} \mathcal{M}^{n-1} \stackrel{\tilde{\delta}}{\longleftarrow} \mathcal{M}^{n-2} \stackrel{\tilde{\delta}}{\longleftarrow} \cdots
$$

has the same cohomology as that of $M$. In section 3, we derive an explicit formula for such a differential in terms of gradient paths of $f$. Let us now be a bit more explicit. A gradient path $\gamma$ of $f$ is a finite sequence of distinct simplices

$$
\gamma: a_{0}^{(p)}, b_{0}^{(p \pm 1)}, a_{1}^{(p)}, b_{1}^{(p \pm 1)}, a_{2}^{(p)}, \ldots
$$

such that for each pair of consecutive simplices, one is a maximal face of the other. Moreover, we require that $f\left(a_{0}\right) \geq f\left(b_{0}\right) \geq f\left(a_{1}\right) \geq f\left(b_{1}\right) \geq \ldots$. We present a slightly different, but equivalent, definition in section 1.) If the final simplex in the gradient path $\gamma$ above is $a_{r}^{(p)}$, then we say that $\gamma$ has length $r$. If it ends with $b_{r}^{(p \pm 1)}$, then we say that $\gamma$ has length $r \frac{1}{2}$. For any simplices $a$ and $b$, let $\Gamma(a, b)$ denote the set of gradient paths from $a$ to $b$ (of any length), i.e., such that the first simplex in the sequence is $a$ and the last simplex in the sequence is $b$. If we wish to indicate the Morse function $f$, we will write $\Gamma_{f}(a, b)$. If $a$ is an oriented simplex of $M$, we will let $a^{*}$ denote the dual cosimplex. That is, if $b$ is an oriented simplex of $M$, then

$$
a^{*}(b)=\left\{\begin{aligned}
1 & \text { if } a=b, \\
-1 & \text { if } a=-b \\
0 & \text { otherwise }
\end{aligned}\right.
$$

We are now ready to present a formula for the differential $\tilde{\delta}$. In Theorem 0.2 we introduce a convention we will follow for the rest of this paper. General simplices of $M$ will be labelled with the lower case Roman letters $a$ and $b$, occasionally with subscripts. Simplices which are critical for the Morse function under discussion will be labelled with the capital Roman letters $A$ and $B$. 
Theorem 0.2. Choose an orientation for each critical simplex of $f$, and write, for a critical p-simplex $A$,

$$
\tilde{\delta}\left(A^{*}\right)=\sum_{\text {critical } B^{(p+1)}} D_{A, B} B^{*}
$$

Then

$$
D_{A, B}=\sum_{\gamma \in \Gamma(B, A)} m(\gamma)
$$

where $m(\gamma)= \pm 1$ is determined by whether or not the orientations of $A$ and $B$ agree relative to $\gamma$. (This is all made precise in section 1.)

That is, the differential $\tilde{\delta}$ is determined by simply counting the gradient paths from one critical simplex to another. It is not hard to see that if $A$ and $B$ are critical and if $\operatorname{dimension}(B) \neq \operatorname{dimension}(A)+1$, then there are no gradient paths from $B$ to $A$.

We can express this differential pictorially by Figure 0.1. The line segment labelled with $f$ represents a gradient path of the function $f$. Open dots will represent critical simplices; so the open dots at the endpoints of the segment indicate that we only consider gradient paths from one critical simplex to another. The differential $\tilde{\delta}$ is constructed by counting all configurations of gradient paths in $M$ which match that of the figure.

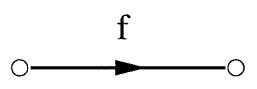

Figure 0.1. The differential

We can now go further. Let $f$ and $g$ be two Morse functions, and $\mathcal{M}^{*}(f)$ and $\mathcal{M}^{*}(g)$ the corresponding Morse cocomplexes. Using the same ideas, we can construct a map from $\mathcal{M}^{*}(f)$ to $\mathcal{M}^{*}(g)$ that induces the identity map on cohomology.

Theorem 0.3. Choose an orientation for each simplex of $M$. For any two Morse functions $f$ and $g$ on $M$, define a map

$$
\tilde{\mathcal{I}}: \mathcal{M}^{*}(f) \rightarrow \mathcal{M}^{*}(g)
$$

as follows. For any $f$-critical $p$-simplex $A$ set

$$
\tilde{\mathcal{I}}\left(A^{*}\right)=\sum_{g \text {-critical } B^{(p)}} I_{A, B} B^{*},
$$

where

$$
I_{A, B}=\sum_{a} \sum_{\gamma_{1} \in \Gamma_{g}(B, a)} \sum_{\gamma_{2} \in \Gamma_{f}(a, A)} m\left(\gamma_{1}\right) m\left(\gamma_{2}\right)
$$

where the first sum is over all p-simplices a of $M$. Then the map $\tilde{\mathcal{I}}$ induces the identity map on cohomology.

The corresponding diagram for this map is shown in Figure 0.2. Here we count all sequences of simplices in $M$ which can be described as a $g$-gradient path beginning at a critical simplex of $g$ and ending at a simplex $a$, followed by an $f$-gradient path 


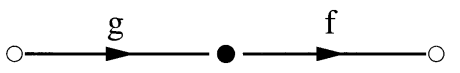

Figure 0.2. The identity map

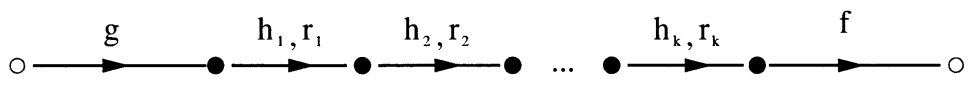

Figure 0.3. Another cochain map that induces the identity map

beginning at $a$ and ending at a critical simplex of $f$. The simplex $a$ is permitted to be any simplex of $M$ (indicated by the solid dot).

The same ideas can be used to construct a number of different Morse cochain maps which induce the identity map on cohomology, with the map described in Theorem 0.3 being the simplest. Consider the diagram shown in Figure 0.3. In this case, rather than having a single solid dot in the line segment, we have a number of solid dots. Each subinterval bounded by two solid dots is labeled by a Morse function $h_{i}$ and a number $r_{i} \in \mathbb{Z}_{\geq 0} \cup\{\infty\}$. This diagram indicates that we should count all sequences of simplices in $M$ which can be described as a $g$-gradient path beginning at a critical simplex of $g$, followed by an $h_{1}$-gradient path of $c$-length $r_{1}$ (this is explained below), followed by an $h_{2}$-gradient path of $c$-length $r_{2}, \ldots$, followed by an $h_{k}$-gradient path of $c$-length $r_{k}$, followed by an $f$-gradient path that ends at an $f$-critical simplex. Each transition can occur at any simplex of $M$. For any $r \in \mathbb{Z}_{\geq 0} \cup \infty$, a gradient path is said to have $c$-length $r$ if either it has length $r$ or it contains a critical simplex and has integer length $\leq r$. For any discrete Morse function $f$, any simplices $a, b$, and any $r \in \mathbb{Z}_{\geq 0} \cup \infty$, let $\Gamma_{f}^{(r)}(a, b)$ denote the set of all $f$-gradient paths of $c$-length $r$ from $a$ to $b$. Note that if either $a$ or $b$ is critical, then $\Gamma_{f}^{(\infty)}(a, b)$ is the set of all integer length $f$-gradient paths from $a$ to $b$.

This notion of $c$-length may seem strange, but in fact is a natural combinatorial analogue of a phenomenon that arises in smooth Morse theory. Suppose one flows down the gradient of a smooth Morse function for time $r$. The issue is the relationship between the time $r$ and the length of the corresponding flowline. If the flowline does not go near a critical point, then its length is at most a bounded (multiplicative) factor away from $r$. On the other hand, if the flowline goes near a critical point, then its length is bounded above by a multiplicative factor of $r$, but it may be as small as we like. Thus, a gradient path of $c$-length $r$ can be thought of as a discrete analogue of flowing for time $r$.

We now give a precise description of the map indicated by Figure 0.3 in the following theorem.

Theorem 0.4. Choose an orientation for every simplex of $M$. For any Morse functions $f, g, h_{1}, h_{2}, \ldots, h_{k}$, and any $r_{1}, r_{2}, \ldots, r_{k} \in \mathbb{Z}_{\geq 0} \cup\{\infty\}$, define a map

$$
\tilde{\mathcal{I}}: \mathcal{M}^{*}(f) \rightarrow \mathcal{M}^{*}(g)
$$

as follows. For any $f$-critical simplex $A$ set

$$
\tilde{\mathcal{I}}\left(A^{*}\right)=\sum_{g \text {-criticalB }(p)} I_{A, B} B^{*}
$$


where

$$
\begin{aligned}
I_{A, B}= & \sum_{a_{1}, a_{2}, a_{3}, \ldots, a_{k}, a_{k+1}} \sum_{\gamma_{0} \in \Gamma_{g}^{(\infty)}\left(B, a_{1}\right)} \sum_{\gamma_{k} \in \Gamma_{h_{k}}^{\left(r_{k}\right)} \Gamma_{h_{1}}^{\left(r_{1}\right)}\left(a_{k}, a_{k+1}\right)} \sum_{\gamma_{k+1} \in \Gamma_{f}^{(\infty)}\left(a_{2}\right)} \sum_{\gamma_{2} \in \Gamma_{h_{2}}^{\left(r_{2}\right)}\left(a_{2}, a_{3}\right)} m \\
& m\left(\gamma_{1}\right) m\left(\gamma_{2}\right) \ldots m\left(\gamma_{k}\right) m\left(\gamma_{k+1}\right),
\end{aligned}
$$

where the first sum is over all $(k+1)$-tuples $a_{1}, a_{2}, \ldots, a_{k}, a_{k+1}$, of simplices of $M$, and $\Gamma_{h}^{(r)}(a, b)$ denotes the set of h-gradient paths from a to $b$ of $c$-length $r$. Then the map $\tilde{\mathcal{I}}$ induces the identity map on cohomology.

Now let $f_{1}, f_{2}$, and $g$ be Morse functions on $M$. Our next goal is to describe a cochain map

$$
\widetilde{U}: \mathcal{M}^{*}\left(f_{1}\right) \otimes \mathcal{M}^{*}\left(f_{2}\right) \rightarrow \mathcal{M}^{*}(g)
$$

that induces the cup product on cohomology. Choose an ordering of the vertices of $M$. This ordering allows one to define a map

$$
\cup: C^{*}(M, \mathbb{Z}) \otimes C^{*}(M, \mathbb{Z}) \rightarrow C^{*}(M, \mathbb{Z})
$$

on cochains that induces the cup product on cohomology (see section 5 for details).

Theorem 0.5. Choose an orientation for every simplex of $M$. For any Morse functions $f_{1}, f_{2}$, and $g$, define a map

$$
\widetilde{\cup}: \mathcal{M}^{*}\left(f_{1}\right) \otimes \mathcal{M}^{*}\left(f_{2}\right) \rightarrow \mathcal{M}^{*}(g)
$$

as follows. For any $f_{1}$-critical $p$-simplex $A_{1}$, any $f_{2}$-critical $q$-simplex $A_{2}$, set

$$
\widetilde{\cup}\left(A_{1}^{*} \otimes A_{2}^{*}\right)=\sum_{g \text {-criticalB }} \widetilde{\cup}_{A_{1} \otimes A_{2}, B} B^{*},
$$

where

$$
\begin{gathered}
\widetilde{\cup}_{A_{1} \otimes A_{2}, B}=\sum_{\sum_{a_{1}^{*} \cup \Gamma_{2}^{*}= \pm b^{*}}}\left\langle a_{1}^{*} \cup a_{2}^{*}, b^{*}\right\rangle \\
\sum_{f_{1}\left(a_{1}, A_{1}\right)} \sum_{\gamma_{2} \in \Gamma_{f_{2}}^{(\infty)}\left(a_{2}, A_{2}\right)} \sum_{\gamma \in \Gamma_{g}^{(\infty)}(B, b)} m\left(\gamma_{1}\right) m\left(\gamma_{2}\right) m(\gamma)
\end{gathered}
$$

where the first sum is over all triples $a_{1}, a_{2}, b$ of simplices in $M$ such that $a_{1}^{*} \cup a_{2}^{*}=$ $\pm b^{*}$. Then the map $\widetilde{\cup}$ induces the cup product map on cohomology.

In this case, the appropriate diagram is shown in Figure 0.4. Here, we are counting triples consisting of a gradient path $\gamma$ from a critical simplex of $g$ to a simplex $b$, a gradient path $\gamma_{1}$ from a simplex $a_{1}$ to a critical simplex of $f_{1}$, and a gradient path $\gamma$ from a simplex $a_{2}$ to a critical simplex of $f_{2}$, such that $a_{1}^{*} \cup a_{2}^{*}= \pm b^{*}$. In particular, in such a diagram, when we see one line segment coming into a solid dot and two lines coming out, that means that the two outgoing gradient paths begin at simplices which have the property that the cup product of their duals is \pm the dual of the endpoint of the incoming gradient path. If we have chosen a cup product on cochains which is not commutative, then we must also indicate an ordering for the two outgoing lines.

Just as in the case of the identity map, we can construct other maps between the Morse cochain complexes that induce the cup product by subdividing the edges 


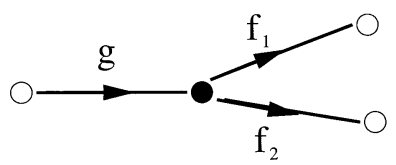

Figure 0.4. The cup product

of the diagram in Figure 0.4 using solid dots. Just as before, to each edge bounded by two solid dots we must assign a choice of a Morse function and a nonnegative integer. For example, the graph shown in Figure 0.5 corresponds to the cochain map $P: \mathcal{M}^{*}(f) \otimes \mathcal{M}^{*}\left(f_{2}\right) \rightarrow \mathcal{M}^{*}(g)$ defined as follows. For any $f_{1}$-critical $p$-simplex $A_{1}$, any $f_{2}$-critical $q$-simplex $A_{2}$, set

$$
P\left(A_{1}^{*} \otimes A_{2}^{*}\right)=\sum_{g \text {-criticalB }} P_{A_{1} \otimes A_{2}, B} B^{*},
$$

where

$$
\begin{gathered}
P_{A_{1} \otimes A_{2}, B}=\sum_{\substack{a_{1}, a_{2}, b \\
\text { s.t. } a_{1}^{*} \cup a_{2}^{*}= \pm b^{*}}}\left\langle a_{1} \cup a_{2}, b\right\rangle \sum_{a_{3}, a_{4}, a_{5}, b_{1}} \sum_{\gamma_{0} \in \Gamma_{g}^{(\infty)}\left(B, b_{1}\right)} \sum_{\gamma_{1} \in \Gamma_{h_{1}}^{\left(r_{1}\right)}\left(b_{1}, b\right)} \sum_{\gamma_{2} \in \Gamma_{h_{2}}^{\left(r_{2}\right)}\left(a_{1}, a_{3}\right)} \sum_{\gamma_{3} \in \Gamma_{h_{3}}^{\left(r_{3}\right)}\left(a_{3}, a_{4}\right)} \sum_{\gamma_{4} \in \Gamma_{h_{4}}^{\left(r_{4}\right)}\left(a_{2}, a_{5}\right)} \sum_{\gamma_{5} \in \Gamma_{f_{1}}^{(\infty)}\left(a_{4}, A_{1}\right)} \sum_{\gamma_{6} \in \Gamma_{f_{2}}^{(\infty)}\left(a_{5}, A_{2}\right)} \prod_{i=0}^{6} m\left(\gamma_{i}\right),
\end{gathered}
$$

where the first sum is over all triples $a_{1}, a_{2}, b$ of simplices in $M$ such that $a_{1}^{*} \cup a_{2}^{*}=$ $\pm b^{*}$, and the second sum is over all 4 -tuples of simplices $a_{3}, a_{4}, a_{5}, b_{1}$ in $M$. Then this map $P$ induces the cup product map on cohomology.

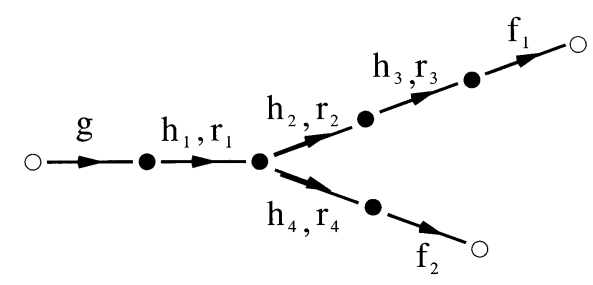

Figure 0.5. Another cochain map that induces the cup product

Two more examples are presented in section 5 . Here we simply present the appropriate diagrams. One can use such ideas to calculate the Euler characteristic. One possible diagram is shown in Figure 0.6(i). As we describe in section 5, this diagram represents, for each $p=0,1,2, \ldots, \operatorname{dim}(M)$, a map from $\mathcal{M}^{p}(g)$ to itself, and the alternating sum of the traces of these maps is the Euler characteristic of $M$. In Figure 0.6(i) we have not drawn the simplest such diagram. We could have left out the edge labelled with the Morse function $h$ and drawn a loop comprised only of the two edges labelled with $f$. However, in that case the formula would tell us to look for all gradient paths of $f$ which form a loop. The only such paths 
are those of length 0 . The resulting formula for the Euler characteristic is simply the alternating sum of the number of critical simplices of $f$ of dimension $p$. The diagram with the additional edge yields a more interesting formula.

Just as in the earlier examples, the edges of this figure can be subdivided, with a Morse function and a nonnegative integer assigned to each edge, and the chain map corresponding to the new diagram will still result in a chain map with the property that the alternating sum of the traces is the Euler characteristic. For example, one can take $f$ to be the trivial Morse function, with respect to which all simplices are critical. If one then subdivides the edge corresponding to the Morse function $h$ (otherwise the resulting formula for Euler characteristic would be the familiar alternating sum of the number of simplices of dimension $p$ ), one learns that for any Morse functions $h_{1}$ and $h_{2}$, and any $r_{1}, r_{2} \in \mathbb{Z}_{\geq 0} \cup\{\infty\}$, the Euler characteristic can be calculated by counting configurations of gradient paths as in Figure 0.6(ii).

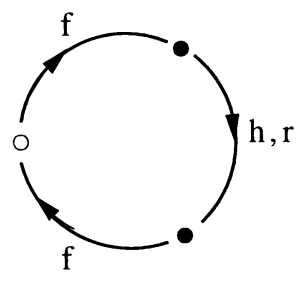

(i)

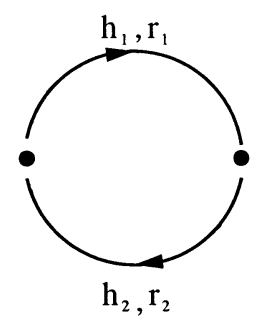

(ii)

Figure 0.6. The Euler characteristic

If $M$ is a connected simplicial complex, then one can consider the fundamental class in $H^{0}(M, \mathbb{Z})$. A 0 -cochain representing this class can be constructed by counting gradient paths in $M$ corresponding to the diagram shown in Figure 0.7(i), with the extra condition that the endpoint be a critical simplex of dimension 0 (i.e., a vertex). In the case that the simplicial complex has additional structure, one can take the dual point of view. For example, if $M$ is an orientable pseudo-manifold of dimension $n$, then one can choose a generator of $H_{n}(M, \mathbb{Z})$. An $n$-chain in the Morse complex which represents this class can be constructed by counting gradient paths in $M$ corresponding to the diagram shown in Figure 0.7(ii), with the extra condition that the source be a critical $n$-simplex. If $M$ is not orientable, then the same holds true, but one must work with coefficients in $\mathbb{Z}_{2}$. This is explained in section 5 .

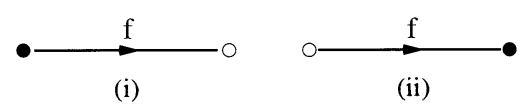

Figure 0.7. The fundamental class

Other examples of the Morse theory approach to cohomology operations can be found in references [2] and [11, 12]. In particular, the authors show how one can 
view the Massey product and the Steenrod square operation from this point of view.

It is interesting to note that in the classical setting of smooth Morse theory, one often restricts attention to a generic Morse function, i.e., a Morse-Smale function, or a generic set of Morse functions, so that the set of gradient paths, or configurations of gradient paths, that one is to count will be finite and nondegenerate (i.e., have a well-defined multiplicity). In the discrete setting there are no such concerns. In the examples shown in this introduction, any set of discrete Morse functions may be chosen in order to construct the appropriate maps. The Morse functions need not be distinct. In particular, one may set each Morse function to be the trivial Morse function with respect to which all simplices are critical. In this case the maps constructed are the usual maps on cochains.

It is interesting to compare the discrete Morse theory constructions we will present here with the analogous constructions in smooth Morse theory. The gradient paths we consider here are the discrete analogues of the downward flowlines of the gradient of a smooth Morse function on a smooth Riemannian manifold. In section 1 we will introduce a gradient flow map $\Phi$, by which, loosely speaking, a simplex flows along the gradient paths. This leads to a map $\Phi^{\infty}$, the result of flowing for infinite time, which takes a critical simplex $A$ to a chain $\Phi^{\infty}(A)$ which is invariant under the flow. This chain $\Phi^{\infty}(A)$ plays the role of the unstable (or descending) cell associated to a critical point of a smooth Morse function. In section 3 we examine the dual picture, and we will introduce the coflow map $\Phi^{*}$ which flows cochains up the Morse function. If $A$ is a critical simplex, then $\left(\Phi^{*}\right)^{\infty}\left(A^{*}\right)$ is a cochain which is invariant under the coflow and plays the role of the stable (or ascending) cell associated to a critical point of a smooth Morse function.

This paper is organized as follows. In sections 1 and 2, we review the construction of the Morse complex in this discrete setting. This is a differential complex built from the critical points of a Morse function which has the same homology as the underlying complex $M$. The differential of this complex is defined in terms of the gradient paths from one critical point to another. Our presentation here is largely self-contained, but a more detailed presentation appears in [5. We begin in section 1 with a precise description of the gradient paths, the gradient vector field, and the gradient flow operator associated to a discrete Morse function. In section 2 we piece these together to construct the Morse complex. In section 3 we show how one can define a dual complex, which we call the Morse cocomplex, to calculate the cohomology of $M$. We also show how, given a cohomology class, one can find a cochain in the Morse cocomplex which represents this cohomology class. In section 4 we show that for any map $L$ on cohomology, induced by a map $\mathcal{L}$ on cochains, there is a map on Morse cochains, that is, cochains in the Morse cocomplex, which induces $L$. Moreover, we give an explicit formula for the map. Lastly, in section 5, we apply the formulas found in section 4 to derive the explicit maps described in the introduction and illustrated in Figures 0.1-0.7.

\section{Gradient Vector Fields and the Corresponding Flow}

In this section we review the definitions and basic properties of the gradient vector field, gradient paths, and the flow along the gradient. Most of the results in this section appeared in [5]. The presentation here is slightly different than that in [5] to allow for more general applications. 
Let $M$ be a finite simplicial complex, along with a Morse function $f$. Let $V$ denote the set of pairs $\left\{a^{(p)}<b^{(p+1)}\right\}$ of simplices of $M$ which satisfy $f(a) \geq f(b)$. These pairs are pairwise disjoint. We call $V$ the gradient vector field of $f$. In fact, there is no need to introduce $f$ at all, as all of the relevant information is contained in $V$. Note that the critical simplices of $f$ are precisely the simplices of $M$ which are not contained in any pair in $V$.

It is useful to consider the gradient vector field as a map of chains,

$$
V: C_{*}(M, \mathbb{Z}) \rightarrow C_{*}(M, \mathbb{Z}),
$$

rather than a collection of pairs. If $\left\{a^{(p)}<b^{(p+1)}\right\}$ satisfy $f(a) \geq f(b)$, then set $V(a)= \pm b$ with the sign chosen so that $\langle a, \partial V(b)\rangle=-1$. We define $V(a)$ to be 0 for all simplices $a$ for which there is no such $b$. We now extend $V$ linearly to a map

$$
V: C_{p}(M, \mathbb{Z}) \rightarrow C_{p+1}(M, \mathbb{Z})
$$

for each $p$.

Any such map $V$ that arises from a Morse function will be called a gradient vector field. Thinking of $V$ as a collection of pairs $\left\{a^{(p)}<b^{(p+1)}\right\}$, we see that every simplex $a$ of $M$ satisfies exactly one of the following:

(i) $a$ is the smaller simplex in one such pair,

(ii) $a$ is the larger simplex in one such pair,

(iii) $a$ is critical.

Thinking of $V$ as a map on chains, this fact translates into the following statement.

Lemma 1.1. If $V: C_{*}(M, \mathbb{Z}) \rightarrow C_{*}(M, \mathbb{Z})$ is a gradient vector field, then

(i) $V^{2}=0$,

and

(ii) for each $p, V$ induces a partition of the p-simplices of $M$

$$
\begin{aligned}
& \{p \text {-simplices of } M\} \\
& =\left\{a^{(p)} \text { such that } V(a) \neq 0\right\} \\
& \quad \cup\left\{a^{(p)} \text { such that } a \in \operatorname{Image}(V)\right\} \cup\left\{a^{(p)} \text { such that } a \text { is critical }\right\} .
\end{aligned}
$$

A gradient path is a sequence of simplices (satisfying certain properties that we will describe shortly) along which the function $f$ is decreasing. Such a sequence should be thought of as the combinatorial analogue of a flowline of the negative of the gradient of a smooth Morse function on a smooth manifold. Our definition here is slightly different than that presented in the introduction. In particular, a gradient path as we have defined the notion here corresponds to a gradient path of integer length as defined in the introduction.

The building blocks of gradient paths are gradient steps. An upper gradient step $s$ is a sequence of simplices

$$
s: a_{0}^{(p)}, b_{0}^{(p+1)}, a_{1}^{(p)},
$$

where $a_{0}$ and $a_{1}$ are faces of $b_{0}$, and $f\left(a_{0}\right) \geq f\left(b_{0}\right)>f\left(a_{1}\right)$. Equivalently, we require that $a_{0}$ and $a_{1}$ be distinct faces of $b_{0}$, and $V\left(a_{0}\right)= \pm b_{0}$. If $a_{0}$ and $a_{1}$ are oriented simplices, then we define the (algebraic) multiplicity of $s$, denoted by $m(s)$, to be \pm 1 depending on whether or not the chosen orientation on $a_{0}$ induces the chosen orientation on $a_{1}$. More explicitly, choose an orientation for $b_{0}$ and set

$$
m(s)=-\left\langle a_{0}, \partial b_{0}\right\rangle\left\langle\partial b_{0}, a_{1}\right\rangle .
$$


Note that $m(s)$ is independent of the chosen orientation for $b_{0}$. A lower gradient step $s$ is a sequence of simplices

$$
s: a_{0}^{(p)}, b_{0}^{(p-1)}, a_{1}^{(p)},
$$

where $b_{0}$ is a face of both $a_{0}$ and $a_{1}$, and $f\left(a_{0}\right)>f\left(b_{0}\right) \geq f\left(a_{1}\right)$. Equivalently, we require that $b_{0}$ is a face of both $a_{0}$ and $a_{1}, a_{0} \neq a_{1}$, and $V\left(b_{0}\right)= \pm a_{1}$. If $a_{0}$ and $a_{1}$ are oriented simplices, then we define the multiplicity of $s$ to be

$$
m(s)=-\left\langle\partial a_{0}, b_{0}\right\rangle\left\langle b_{0}, \partial a_{1}\right\rangle .
$$

A sequence of simplices $s=a_{0}^{(p)}, b_{0}^{(p \pm 1)}, a_{1}^{(p)}$ is called a gradient step if it is either an upper or lower gradient step.

A gradient path $\gamma$ from a $p$-simplex $a$ to a $p$-simplex $a^{\prime}$ is a sequence of gradient steps $\gamma=s_{1}, s_{2}, \ldots, s_{r}$, such that $s_{1}$ begins with $a, s_{r}$ ends with $a^{\prime}$, and, for each $i=1,2, \ldots, r-1, s_{i+1}$ begins where $s_{i}$ ends. That is, $\gamma$ has the form

$$
\gamma: a=a_{0}^{(p)}, b_{0}^{(p \pm 1)}, a_{1}^{(p)}, b_{1}^{(p \pm 1)}, \ldots, a_{r-1}^{(p)}, b_{r-1}^{(p \pm 1)}, a_{r}^{(p)}=a^{\prime},
$$

where, for each $i=1,2, \ldots, r, s_{i}=a_{i-1}, b_{i-1}, a_{i}$ is a gradient step. We define the length of $\gamma$ to be $r$. If each of the steps $s_{i}$ is an upper (resp. lower) gradient step, then we say that $\gamma$ is an upper (resp. lower) gradient path. If $a$ and $a^{\prime}$ are oriented simplices, we define the multiplicity of $\gamma$ to be

$$
m(\gamma)=\prod_{i=1}^{r} m\left(s_{i}\right)
$$

The following lemma follows immediately from the definitions.

Lemma 1.2. Let $\gamma=s_{1}, s_{2}, \ldots, s_{r}$ and $\gamma^{\prime}=s_{1}^{\prime}, s_{2}^{\prime}, \ldots, s_{r^{\prime}}^{\prime}$ be gradient paths, and suppose that the last simplex in $\gamma$ is equal to the first simplex in $\gamma^{\prime}$. Then $\gamma \circ \gamma^{\prime}=$ $s_{1}, s_{2}, \ldots, s_{r}, s_{1}^{\prime}, s_{2}^{\prime}, \ldots, s_{r^{\prime}}^{\prime}$ is a gradient path, and $m\left(\gamma \circ \gamma^{\prime}\right)=m(\gamma) m\left(\gamma^{\prime}\right)$.

For convenience, we say that for any simplex $a$, the sequence consisting of just the single simplex $a$ is a gradient path from $a$ to itself, having length 0 and multiplicity 1 . By a nontrivial gradient path we will mean any gradient path with length $\geq 1$.

We pause here to introduce some notation that will play a prominent role in the rest of the paper. For any $p$-simplices $a$ and $a^{\prime}$, we let $\Gamma\left(a, a^{\prime}\right)$ denote the set of all gradient paths from $a$ to $a^{\prime}$. On occasion, it will be important to examine some subsets of $\Gamma\left(a, a^{\prime}\right)$. Let $\bar{\Gamma}\left(a, a^{\prime}\right) \subseteq \Gamma\left(a, a^{\prime}\right)\left(\operatorname{resp} . \underline{\Gamma}\left(a, a^{\prime}\right) \subseteq \Gamma\left(a, a^{\prime}\right)\right)$ denote the set of all upper (resp. lower) gradient paths. For any nonnegative integer $r$, we let $\Gamma^{r}\left(a, a^{\prime}\right) \subseteq \Gamma\left(a, a^{\prime}\right)$ (resp. $\left.\Gamma^{\leq r}\left(a, a^{\prime}\right) \subseteq \Gamma\left(a, a^{\prime}\right)\right)$ denote the gradient paths of length $r$ (resp. $\leq r)$ from $a$ to $a^{\prime}$. Lastly, we let $c \Gamma\left(a, a^{\prime}\right) \subseteq \Gamma\left(a, a^{\prime}\right)$ denote the gradient paths which include at least one critical simplex, and $n \Gamma\left(a, a^{\prime}\right) \subseteq \Gamma\left(a, a^{\prime}\right)$ those that do not contain any critical simplices. We will occasionally combine the notation, such as $c \bar{\Gamma}^{r}\left(a, a^{\prime}\right)$, and we hope that the meaning will be clear.

We will now describe the basic properties of gradient paths.

Lemma 1.3. (i) Let $\gamma=s_{0}, s_{1}, s_{2}, \ldots s_{r-1}$ be a gradient path, where each $s_{i}$ is a gradient step. If, for some $k, s_{k}$ is a lower gradient step, then for each $\ell>k, s_{\ell}$ is a lower gradient step.

(ii) If $V(a)=0$, then every nontrivial gradient path beginning at a is a lower gradient path.

(iii) If $a^{\prime}$ is not in the image of $V$, then every gradient path ending at $a^{\prime}$ is an upper gradient path. 
(iv) If $a \neq a^{\prime}$ are critical simplices, then there are no gradient paths from a to $a^{\prime}$.

Proof. (i) A lower gradient step always ends at a simplex $a$ in the image of $V$. An upper gradient step must begin at a simplex $a$ satisfying $V(a) \neq 0$. From Lemma 1.1 we see that there are no simplices $a$ which satisfy both conditions.

(ii) If $V(a)=0$, then any gradient step beginning at $a$ must be a lower gradient step. Now apply (i).

(iii) If $a^{\prime}$ is not in the image of $V$, then any gradient step which ends at $a^{\prime}$ must be an upper gradient step. Now apply (i).

(iv) A simplex $a$ is critical if and only if $V(a)=0$, and $a$ is not in the image of $V$. Now apply (ii) and (iii).

We now define the (discrete time) gradient flow map. The gradient flow

$$
\Phi: C_{*}(M, \mathbb{Z}) \rightarrow C_{*}(M, \mathbb{Z})
$$

is defined by the formula

$$
\Phi=1+\partial \circ V+V \circ \partial,
$$

where $\partial$ denotes the standard boundary map on chains. Note that for each $p, \Phi$ restricts to a map $\Phi: C_{p}(M, \mathbb{Z}) \rightarrow C_{p}(M, \mathbb{Z})$. Our goal now is to give an explicit description of the map $\Phi$ in terms of gradient paths.

Choose an orientation for each simplex of $M$. We can then express $\Phi$ in coordinates by setting, for any $p$-simplex $a$,

$$
\Phi(a)=\sum_{b^{(p)}} F(a, b) b .
$$

More generally, for any nonnegative integer $r$, we set

$$
\Phi^{r}(a)=\sum_{b^{(p)}} F^{r}(a, b) b .
$$

Note that $F^{r}(a, b) \neq(F(a, b))^{r}$.

The following theorem is straighforward to verify and follows from Theorem 6.6 in [5].

Theorem 1.4. If $a$ is not a critical simplex, then for any simplex $b$,

$$
F(a, b)=\sum_{\gamma \in \Gamma^{1}(a, b)} m(\gamma) .
$$

If $a$ is a critical simplex, then for any simplex $b$,

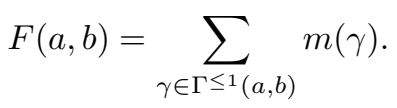

Combining this theorem with Lemma 1.2 allows us to give a similar expression for $F^{r}(a, b)$.

Theorem 1.5. For any p-simplices $a$ and $b$,

$$
F^{r}(a, b)=\sum_{\gamma \in n \Gamma^{r}(a, b)} m(\gamma)+\sum_{\gamma \in c \Gamma \leq r(a, b)} m(\gamma) .
$$


The notation $\Gamma^{(r)}$ was presented in the introduction. The relation to our current notation is

$$
\Gamma^{(r)}(a, b)=n \Gamma^{r}(a, b) \cup c \Gamma^{\leq r}(a, b)
$$

so that

$$
F^{r}(a, b)=\sum_{\gamma \in \Gamma^{(r)}(a, b)} m(\gamma) .
$$

Corollary 1.6. There is an $N$ such that $\Phi^{N}=\Phi^{N+1}=\cdots=\Phi^{\infty}$. If we write

$$
\Phi^{\infty}(a)=\sum_{b^{(p)}} F^{\infty}(a, b) b,
$$

then for any $p$-simplices $a$ and $b$,

$$
F^{\infty}(a, b)=\sum_{\gamma \in c \Gamma(a, b)} m(\gamma) .
$$

Of particular significance is the case when one of the endpoints is a critical simplex. For future reference, we state this case separately.

Corollary 1.7. If either $a$ or $b$ is critical, then

$$
F^{\infty}(a, b)=\sum_{\gamma \in \Gamma(a, b)} m(\gamma) .
$$

If a is critical, then we can also write this as

$$
F^{\infty}(a, b)=\sum_{\gamma \in \underline{\Gamma}(a, b)} m(\gamma) .
$$

If $b$ is critical, then

$$
F^{\infty}(a, b)=\sum_{\gamma \in \bar{\Gamma}(a, b)} m(\gamma)
$$

Corollary 1.8. Let a be a p-simplex and $r$ a positive integer.

(i) If a is not critical, then $\Phi^{r}(a)$ is supported on p-simplices b that satisfy $f(b)<$ $f(a)$.

(ii) If a is critical, then $\Phi^{r}(a)=a+c$ where the $p$-chain $c$ is supported on simplices $b$ that are in the image of $V$ and that satisfy $f(b)<f(a)$.

\section{Morse Homology}

Let $M$ be a finite simplicial complex of dimension $n$ and $V$ a gradient vector field on $M$. In this section we review how to construct a differential complex from the critical points and the gradient paths of $V$ which has the same homology as $M$. The details appear in section 7 of [5].

Our main goal here is to understand the homology of $M$, or equivalently, the homology of the simplicial chain complex

$$
C_{*}: 0 \longrightarrow C_{n}(M, \mathbb{Z}) \stackrel{\partial}{\longrightarrow} C_{n-1}(M, \mathbb{Z}) \stackrel{\partial}{\longrightarrow} C_{n-2}(M, \mathbb{Z}) \stackrel{\partial}{\longrightarrow} \cdots .
$$

Let $C_{p}^{\Phi} \subseteq C_{p}(M, \mathbb{Z})$ denote the $\Phi$-invariant $p$-chains, i.e., those $p$-chains $c$ such that $\Phi(c)=c$. Since

$$
\partial \circ \Phi=\Phi \circ \partial
$$


the $\Phi$-invariant chains form a subcomplex

$$
\mathcal{C}^{\Phi}: 0 \longrightarrow C_{n}^{\Phi} \stackrel{\partial}{\longrightarrow} C_{n-1}^{\Phi} \stackrel{\partial}{\longrightarrow} C_{n-2}^{\Phi} \stackrel{\partial}{\longrightarrow} \cdots
$$

The crucial fact is the following theorem.

Theorem 2.1. $H_{*}\left(\mathcal{C}^{\Phi}\right)=H_{*}(M, \mathbb{Z})$.

That is, this complex, the discrete Morse complex, has the same homology as the underlying space. The proof of this theorem is not difficult. To prove that the stabilization map $\Phi^{\infty}: C_{*}(M, \mathbb{Z}) \rightarrow C_{*}^{\Phi}$ induces an isomorphism on homology (with the inverse isomorphism induced by the natural injection), it is sufficient to find an algebraic homotopy operator, i.e., an operator $K: C_{*}(M, \mathbb{Z}) \rightarrow C_{*+1}(M, \mathbb{Z})$ such that $\Phi^{\infty}-1=\partial \circ K+K \circ \partial$. From Corollary 1.6] we know that $\Phi^{\infty}=\Phi^{N}$ for some $N$. If $N=1$, then $K=V$ works. The general case is not much harder.

Our next goal is to describe this Morse complex in terms of critical simplices. For each $p$, let $\mathcal{M}_{p} \subseteq C_{p}(M, \mathbb{Z})$ denote the span of the critical $p$-simplices. We then have a natural map

$$
\Phi^{\infty}: \mathcal{M}_{p} \rightarrow C_{p}^{\Phi}
$$

There is also a natural projection

$$
\pi_{\mathcal{M}}: C_{p}(M, \mathbb{Z}) \rightarrow \mathcal{M}_{p}
$$

defined by simply restricting to the critical simplices. That is, if $c=\sum_{a} c_{a} a$ is a chain, where the sum is over oriented simplices $a$, set

$$
\pi_{\mathcal{M}}(c)=\sum_{\text {critical } a} c_{a} a
$$

Theorem 2.2. For each $p$, the map $\Phi^{\infty}: \mathcal{M}_{p} \rightarrow C_{p}^{\Phi}$ is an isomorphism. The inverse is given by the map $\pi_{\mathcal{M}}: C_{p}^{\Phi} \rightarrow \mathcal{M}_{p}$.

Proof. It follows from Corollary 1.7 and Lemma 1.3 (iv) that

$$
\pi_{\mathcal{M}} \circ \Phi^{\infty}: \mathcal{M}_{p} \rightarrow \mathcal{M}_{p}
$$

is the identity map, and hence $\pi_{\mathcal{M}}: C_{p}^{\Phi} \rightarrow \mathcal{M}_{p}$ is surjective. To see that this map is injective it is sufficient to see that if $c \in C_{p}^{\Phi}$ satisfies $\pi_{\mathcal{M}}(c)=0$, then $c=0$. This follows from Corollary 1.8(i).

Thus, the Morse complex can be defined equivalently as the complex

$$
\mathcal{M}: 0 \longrightarrow \mathcal{M}_{n} \stackrel{\tilde{\partial}}{\longrightarrow} \mathcal{M}_{n-1} \stackrel{\tilde{\partial}}{\longrightarrow} \mathcal{M}_{n-2} \stackrel{\tilde{\partial}}{\longrightarrow} \cdots
$$

where $\widetilde{\partial}=\pi_{\mathcal{M}} \circ \partial \circ \Phi^{\infty}$ is the differential induced by the above isomorphism. This differential can be described more explicitly in terms of gradient paths from one critical simplex to another.

Theorem 2.3. Choose an orientation for each critical simplex. Then for any critical simplex $B^{(p+1)}$,

$$
\widetilde{\partial} B=\sum_{\text {critical } A^{(p)}}\left[\sum_{a^{(p)}<B}\langle\partial B, a\rangle F^{\infty}(a, A)\right] A .
$$


Proof. Let $B^{p+1}$ be a critical simplex. To determine $\tilde{\partial} B$ we use the formula $\tilde{\partial}=$ $\pi_{\mathcal{M}} \circ \partial \circ \Phi^{\infty}=\pi_{\mathcal{M}} \circ \Phi^{\infty} \circ \partial$, so that

$$
\begin{gathered}
\tilde{\partial} B=\sum_{\text {critical } A^{(p)}}\left\langle\Phi^{\infty}(\partial B), A\right\rangle A \\
=\sum_{\text {critical } A^{(p)}}\left[\sum_{a^{(p)}<B}\langle\partial B, a\rangle\left\langle\Phi^{\infty}(a), A\right\rangle\right] A \\
=\sum_{\text {critical } A^{(p)}}\left[\sum_{a^{(p)}<B}\langle\partial B, a\rangle F^{\infty}(a, A)\right] A .
\end{gathered}
$$

Note that this description of the differential is slightly different from the description in the introduction that was illustrated in Figure 0.1. However, these definitions are equivalent. Theorem 2.3 tells us to count all sequences beginning at $B^{(p+1)}$ and followed by an integer length gradient path $\gamma$ from $a$ to $A$, for some $a^{(p)}<B$. Such a sequence is precisely the sort of half-integer length gradient path described in the introduction. Conversely, any half-integer length gradient path from $B$ to $A$, as defined in the introduction, is of the form $B$ followed by an integer length gradient path from $a$ to $A$, for some $a^{(p)}<B$.

\section{Morse Cohomology}

To create a presentation of cohomology from the point of view of discrete Morse theory, one need only take the presentation of homology in the previous section and reverse all of the arrows, taking the duals of all of the appropriate spaces and operators. This is essentially what we will do in this section.

As in the previous sections, we let $M$ denote a finite simplicial complex. By a cosimplex, we mean an element of the basis of $C^{*}(M, \mathbb{Z})$ which is dual to the basis of $C_{*}(M, \mathbb{Z})$ consisting of the oriented simplices. That is, if $a$ is an oriented $p$-simplex, then the dual cosimplex $a^{*}$ is defined as in (0.1).

Suppose that $M$ is endowed with a gradient vector field $V$. We say that a cosimplex $a^{*}$ is a critical cosimplex of $V$, if and only if $a$ is a critical simplex of $V$. Let

$$
V^{*}: C^{*}(M, \mathbb{Z}) \rightarrow C^{*-1}(M, \mathbb{Z})
$$

be the dual of the operator $V$. More explicitly, for any $\operatorname{cochain} c^{*}$ and any chain $c$,

$$
\left[V^{*}\left(c^{*}\right)\right](c)=c^{*}(V(c)) \text {. }
$$

Alternately, just as in the case of our definition of $V$, we can begin by defining $V^{*}$ as a map on cosimplices. For any cosimplices $a^{*}$ and $b^{*}, V^{*}\left(a^{*}\right)=b^{*}$ if and only if $V(b)=a$. We then extend $V^{*}$ linearly to all of $C^{*}(M, \mathbb{Z})$. It is easy to check that these definitions of $V^{*}$ are equivalent.

We define the coflow

$$
\Phi^{*}: C^{*}(M, \mathbb{Z}) \rightarrow C^{*}(M, \mathbb{Z})
$$


as the dual of the flow operator $\Phi$. That is, for any cochain $c^{*}$ and any chain $c,\left[\Phi^{*}\left(c^{*}\right)\right](c)=c^{*}(\Phi(c))$. Equivalently, the operator $\Phi^{*}$ is given by the formula $\Phi^{*}=1+\delta \circ V^{*}+V^{*} \circ \delta$.

In this section we study the cohomology of $M$, or equivalently, the cohomology of the simplicial cocomplex

$$
C^{*}: C^{n}(M, \mathbb{Z}) \stackrel{\delta}{\longleftarrow} C^{n-1}(M, \mathbb{Z}) \stackrel{\delta}{\longleftarrow} C^{n-2}(M, \mathbb{Z}) \stackrel{\delta}{\longleftarrow} \cdots .
$$

We let $C_{\Phi}^{p} \subseteq C^{p}(M, \mathbb{Z})$ denote the $p$-cochains which are invariant under the coflow $\Phi^{*}$. The Morse cochain complex is the complex

$$
\mathcal{C}_{\Phi}: C_{\Phi}^{n} \stackrel{\delta}{\longleftarrow} C_{\Phi}^{n-1} \stackrel{\delta}{\longleftarrow} C_{\Phi}^{n-2} \stackrel{\delta}{\longleftarrow} \cdots .
$$

We have the corresponding theorem.

Theorem 3.1. $H^{*}\left(\mathcal{C}_{\Phi}\right) \cong H^{*}(M, \mathbb{Z})$.

The proof is easily adapted from the proof of Theorem 2.1

Let $\mathcal{M}^{p} \subseteq C^{p}(M, \mathbb{Z})$ denote the span of the critical cosimplices, and $\pi_{\mathcal{M}}$ : $C^{p}(M, \mathbb{Z}) \rightarrow \mathcal{M}^{p}$ the natural projection. The following result corresponds to Theorem 2.2 .

Theorem 3.2. The map $\left(\Phi^{*}\right)^{\infty}: \mathcal{M}^{p} \rightarrow C_{\Phi}^{p}$ is an isomorphism. The inverse map is given by $\pi_{\mathcal{M}}: C^{p}(M, \mathbb{Z}) \rightarrow \mathcal{M}^{p}$.

Thus, the Morse cochain complex can be defined equivalently as the complex

$$
\mathcal{M}^{*}: 0 \longrightarrow \mathcal{M}^{n} \stackrel{\tilde{\delta}}{\longleftarrow} \mathcal{M}^{n-1} \stackrel{\tilde{\delta}}{\longleftarrow} \mathcal{M}^{n-2} \stackrel{\tilde{\delta}}{\longleftarrow} \ldots
$$

where $\tilde{\delta}=\pi_{\mathcal{M}} \circ \delta \circ\left(\Phi^{*}\right)^{\infty}$ is the differential induced by the above isomorphism. This differential is described explicitly in the following theorem.

Theorem 3.3. For any oriented critical $p$-cosimplex $A^{*}$,

$$
\tilde{\delta} A^{*}=\sum_{\text {critical } B^{(p+1)}}\left[\sum_{a^{(p)}<B}\langle\partial B, a\rangle F^{\infty}(a, A)\right] B .
$$

This theorem follows from simply taking the dual of the operator $\tilde{\partial}$ that appears in Theorem 2.3 Alternately, one can deduce the formula from Theorem 3.4, which follows from the fact that $\Phi^{*}$ is the dual of the operator $\Phi$.

Theorem 3.4. Choose an orientation for each simplex of $M$, and write, for any simplex $a$ and nonnegative integer $r$,

$$
\left(\Phi^{*}\right)^{r}\left(a^{*}\right)=\sum_{b^{*}}\left(F^{*}\right)^{r}\left(a^{*}, b^{*}\right) .
$$

Then

$$
\left(F^{*}\right)^{r}\left(a^{*}, b^{*}\right)=F^{r}(b, a)
$$

The next two corollaries follow from the analogous results about the operator $\Phi$.

Corollary 3.5. There is an $N$ such that $\left(\Phi^{*}\right)^{N}=\left(\Phi^{*}\right)^{N+1}=\cdots=\left(\Phi^{*}\right)^{\infty}$. If we write

$$
\left(\Phi^{*}\right)^{\infty}\left(a^{*}\right)=\sum_{b^{(p)}}\left(F^{*}\right)^{\infty}\left(a^{*}, b^{*}\right) b^{*}
$$


then for any p-simplices $a$ and $b$,

$$
\left(F^{*}\right)^{\infty}\left(a^{*}, b^{*}\right)=F^{\infty}(b, a) .
$$

Corollary 3.6. Let a be a p-simplex and $r$ a positive integer.

(i) If a is not critical, then $\left(\Phi^{*}\right)^{r}\left(a^{*}\right)$ is supported on $p$-cosimplices $b^{*}$ such that $f(b)>f(a)$.

(ii) If a is critical, then $\left(\Phi^{*}\right)^{r}\left(a^{*}\right)=a^{*}+c^{*}$ where the $p$-cochain $c^{*}$ is supported on cosimplices $b^{*}$ that are in the image of $V^{*}$ and that satisfy $f(b)>f(a)$.

Our next goal is to learn how to find Morse cochains which represent given cohomology classes.

Corollary 3.7. Let $A$ and $B$ be critical p-simplices. Then

$$
\left[\left(\Phi^{*}\right)^{\infty}\left(A^{*}\right)\right]\left(\Phi^{\infty}(B)\right)=\left\{\begin{aligned}
1 & \text { if } A=B, \\
-1 & \text { if } A=-B, \\
0 & \text { otherwise. }
\end{aligned}\right.
$$

Proof. By Corollary 3.6 (ii), $\left(\Phi^{*}\right)^{\infty}\left(A^{*}\right)$ is the sum of $A^{*}$ and $p$-cosimplices in the image of $V^{*}$. By Corollary 1.8 (ii), $\Phi^{\infty}(B)$ is the sum of $B$ and $p$-simplices in the image of $V$. From the partition

$$
\{p \text {-simplices of } M\}=\operatorname{Image}\left(V^{*}\right)+\operatorname{Image}(V)+\{\text { critical p-simplices }\}
$$

we see that

$$
\left[\left(\Phi^{*}\right)^{\infty}\left(A^{*}\right)\right]\left(\Phi^{\infty}(B)\right)=A^{*}(B) .
$$

Theorem 3.8. Suppose that $c^{*} \in C^{*}(M, \mathbb{Z})$ is a cocycle (i.e., is in the kernel of $\delta)$. Let $m^{*}=\pi_{\mathcal{M}}\left(\Phi^{*}\right)^{\infty} c^{*}$. More explicitly, choose an orientation for each simplex of $M$. If $c^{*}=\sum_{a^{*}} c_{a^{*}} a^{*}$, then

$$
m^{*}=\sum_{\text {critical } B^{*}}\left[\sum_{a^{*}} F^{\infty}(B, a) c_{a^{*}}\right] B^{*} .
$$

Then $m^{*} \in \mathcal{M}^{*}$ is a cocycle in the Morse cocomplex and represents the same cohomology class as $c^{*}$.

Proof. The map $\left(\Phi^{*}\right)^{\infty}$ maps $c^{*}$ to a $\Phi^{*}$-invariant cocycle which represents the same cohomology class as $c^{*}$. The map $\pi_{\mathcal{M}}$ is an isomorphism from $C_{\Phi}^{*}$ to $\mathcal{M}^{*}$, and hence takes $\left(\Phi^{*}\right)^{\infty} c^{*}$ to a cocycle in the Morse cocomplex which represents the same cohomology class. Thus

$$
m^{*}=\sum_{\text {critical } B^{*}}\left(\left[\left(\Phi^{*}\right)^{\infty}\left(c^{*}\right)\right](B)\right) B^{*} .
$$

We now observe that for any critical simplex $B$,

$$
\left[\left(\Phi^{*}\right)^{\infty}\left(c^{*}\right)\right](B)=c^{*}\left(\Phi^{\infty}(B)\right)=\sum_{a^{*}} c_{a^{*}} a^{*}\left(\Phi^{\infty}(B)\right)=\sum_{a^{*}} c_{a^{*}} F^{\infty}(B, a) .
$$

Let $h$ be any Morse function on $M$, and let $r$ be in $\mathbb{Z}_{\geq 0} \cup\{\infty\}$. Then for any cocycle $c^{*} \in C^{*}(M, \mathbb{Z}),\left(\Phi_{h}^{*}\right)^{r}\left(c^{*}\right)$ is a cocycle which represents the same cohomology 
class as $c^{*}$. Therefore, in the previous theorem, the cochain $m^{*} \in \mathcal{M}^{*}(f)$ defined by

$$
m^{*}=\pi_{\mathcal{M}}\left(\Phi_{f}^{*}\right)^{\infty}\left(\Phi_{h}^{*}\right)^{r} c^{*}
$$

also satisfies the conclusion of the theorem. More generally, we have the following theorem.

Theorem 3.9. Let $f, h_{1}, h_{2}, \ldots, h_{k}$ be Morse functions on $M$ and $r_{i} \in \mathbb{Z}_{\geq 0} \cup\{\infty\}$, $i=1,2, \ldots, k$. Suppose that $c^{*} \in C^{*}(M, \mathbb{Z})$ is a cocycle. Let

$$
m^{*}=\pi_{\mathcal{M}(f)}\left(\Phi_{f}^{*}\right)^{\infty}\left(\Phi_{h_{1}}^{*}\right)^{r_{1}}\left(\Phi_{h_{2}}^{*}\right)^{r_{2}} \ldots\left(\Phi_{h_{k}}^{*}\right)^{r_{k}} c^{*} .
$$

More explicitly, choose an orientation for each simplex of $M$, write $c^{*}=\sum_{a^{*}} c_{a^{*}} a^{*}$, and let $m^{*}$ denote the sum

$$
\sum_{\text {critical } B^{*}}\left[\sum_{a_{0}^{*}, a_{1}^{*}, a_{2}^{*}, \ldots, a_{k}^{*}} F_{f}^{\infty}\left(B, a_{0}\right) F_{h_{1}}^{r_{1}}\left(a_{0}, a_{1}\right) F_{h_{2}}^{r_{2}}\left(a_{1}, a_{2}\right) \ldots F_{h_{k}}^{r_{k}}\left(a_{k-1}, a_{k}\right) c_{a_{k}^{*}}\right] B^{*},
$$

where the second sum is over all $(k+1)$-tuples of simplices in $M$. Then $m^{*} \in \mathcal{M}^{*}(f)$ is a cocycle in the Morse cocomplex and represents the same cohomology class as $c^{*}$.

\section{Cohomology Operations}

We begin with some general definitions and notation. Suppose that for each $i=1,2, \ldots, r$ we have a differential cocomplex

$$
\mathcal{V}_{i}: V_{i}^{n} \longleftarrow V_{i}^{n-1} \longleftarrow V_{i}^{n-2} \longleftarrow \ldots
$$

with cohomology $H^{*}\left(\mathcal{V}_{i}\right)$. For each $i$ and $j$, let $Z_{i}^{j} \subseteq V_{i}^{j}$ denote the cocycles. For each $z_{i} \in Z_{i}^{*}$ we let $\left[z_{i}\right]$ denote the cohomology class in $H^{*}\left(\mathcal{V}_{i}\right)$ represented by $z_{i}$. More generally, given an element $z \in \bigotimes_{i \in I} Z_{i}^{*}$, we let $[z]$ represent the class in $\bigotimes_{i \in I} H^{*}\left(\mathcal{V}_{i}\right)$ represented by $z$.

Say a multilinear map

$$
L: H^{*}\left(\mathcal{V}_{1}\right) \otimes H^{*}\left(\mathcal{V}_{2}\right) \otimes \cdots \otimes H^{*}\left(\mathcal{V}_{k}\right) \longrightarrow H^{*}\left(\mathcal{V}_{k+1}\right) \otimes H^{*}\left(\mathcal{V}_{k+2}\right) \otimes \cdots \otimes H^{*}\left(\mathcal{V}_{r}\right)
$$

is induced by the multilinear map

$$
\mathcal{L}: V_{1}^{*} \otimes V_{2}^{*} \otimes \cdots \otimes V_{k}^{*} \longrightarrow V_{k+1}^{*} \otimes V_{k+2}^{*} \otimes \cdots \otimes V_{r}^{*}
$$

if the following two properties hold:

(1) $\mathcal{L}\left(Z_{1}^{*} \otimes Z_{2}^{*} \otimes \cdots \otimes Z_{k}^{*}\right) \subseteq\left(Z_{k+1}^{*} \otimes Z_{k+2}^{*} \otimes \cdots \otimes Z_{r}^{*}\right)$

and

(2) for any $z_{i} \in Z_{i}^{*}, i=1,2, \ldots, k$,

$$
\left[\mathcal{L}\left(z_{1} \otimes z_{2} \otimes \cdots \otimes z_{k}\right)\right]=L\left(\left[z_{1}\right] \otimes\left[z_{2}\right] \otimes \cdots \otimes\left[z_{k}\right]\right) .
$$

Now we focus attention on the special case of interest. Let $M$ be a finite simplicial complex and

$$
L: \otimes{ }^{k} H^{*}(M, \mathbb{Z}) \rightarrow \otimes^{\ell} H^{*}(M, \mathbb{Z})
$$

a multilinear map. Suppose that $L$ is induced by the multilinear map

$$
\mathcal{L}: \otimes^{k} C^{*}(M, \mathbb{Z}) \rightarrow \otimes^{\ell} C^{*}(M, \mathbb{Z}) .
$$


Choose an orientation for each simplex of $M$. Then we can express $\mathcal{L}$ in coordinates by writing, for each $a_{1}^{*} \otimes a_{2}^{*} \otimes \cdots \otimes a_{k}^{*} \in \otimes^{k} C^{*}(M, Z)$,

$$
\mathcal{L}\left(a_{1}^{*} \otimes a_{2}^{*} \otimes \cdots \otimes a_{k}^{*}\right)=\sum_{b_{1}^{*}, b_{2}^{*}, \ldots, b_{\ell}^{*}} \mathcal{L}_{a_{1}^{*} \otimes a_{2}^{*} \otimes \cdots \otimes a_{k}^{*}, b_{1}^{*} \otimes b_{2}^{*} \otimes \cdots \otimes b_{\ell}^{*}} b_{1}^{*} \otimes b_{2}^{*}, \otimes \cdots \otimes b_{\ell}^{*}
$$

where the sum is over all $\ell$-tuples $b_{1}^{*}, b_{2}^{*}, \ldots, b_{\ell}^{*}$ of simplices in $M$.

Now let $f_{1}, f_{2}, \ldots, f_{k}$ and $g_{1}, g_{2}, \ldots, g_{\ell}$ be (not necessarily distinct) Morse functions on $M$. Our goal is to find a map

$$
\tilde{\mathcal{L}}: \mathcal{M}^{*}\left(f_{1}\right) \otimes \mathcal{M}^{*}\left(f_{2}\right) \otimes \cdots \otimes \mathcal{M}^{*}\left(f_{k}\right) \longrightarrow \mathcal{M}^{*}\left(g_{1}\right) \otimes \mathcal{M}^{*}\left(g_{2}\right) \otimes \cdots \otimes \mathcal{M}^{*}\left(g_{\ell}\right)
$$

which induces $L$.

All the information needed to find such a formula was provided in the previous sections. Let $z_{i} \in \mathcal{M}_{i}^{*}$ denote the cycles in the corresponding Morse cocomplexes. Then $z_{i}$ corresponds to the cocycle $\left(\Phi_{f_{i}}^{*}\right)^{\infty}\left(z_{i}\right)$ in $C^{*}(M, \mathbb{Z})$. We just need to piece these maps together. For $z=z_{1} \otimes z_{2} \otimes \cdots \otimes z_{k} \in \otimes^{k} C^{*}(M, \mathbb{Z})$, we let $\Phi_{f}^{*}(z)$ denote the element $\Phi_{f_{1}}\left(z_{1}\right) \otimes \Phi_{f_{2}}\left(z_{2}\right) \otimes \cdots \otimes \Phi_{f_{k}}\left(z_{k}\right)$. For $z=z_{1} \otimes z_{2} \otimes \cdots \otimes z_{\ell} \in \otimes^{\ell} C^{*}(M, \mathbb{Z})$, $\Phi_{g}^{*}(z)$ is defined similarly.

The following theorem follows from our work in the previous sections.

Theorem 4.1. The map

$$
\begin{gathered}
\tilde{\mathcal{L}}=\pi_{\mathcal{M}} \circ\left(\Phi_{g}^{*}\right)^{\infty} \circ \mathcal{L} \circ\left(\Phi_{f}^{*}\right)^{\infty}: \mathcal{M}^{*}\left(f_{1}\right) \otimes \mathcal{M}^{*}\left(f_{2}\right) \otimes \cdots \otimes \mathcal{M}^{*}\left(f_{k}\right) \\
\longrightarrow \mathcal{M}^{*}\left(g_{1}\right) \otimes \mathcal{M}^{*}\left(g_{2}\right) \otimes \cdots \otimes \mathcal{M}^{*}\left(g_{\ell}\right)
\end{gathered}
$$

induces $L$.

Expressing the map $\tilde{\mathcal{L}}$ in coordinates, we find the following corollary.

Corollary 4.2. Define a map

$$
\tilde{\mathcal{L}}: \mathcal{M}^{*}\left(f_{1}\right) \otimes \mathcal{M}^{*}\left(f_{2}\right) \otimes \cdots \otimes \mathcal{M}^{*}\left(f_{k}\right) \longrightarrow \mathcal{M}^{*}\left(g_{1}\right) \otimes \mathcal{M}^{*}\left(g_{2}\right) \otimes \cdots \otimes \mathcal{M}^{*}\left(g_{\ell}\right)
$$

by setting, for any critical $A_{i}^{*} \in \mathcal{M}^{*}\left(f_{i}\right), i=1,2, \ldots, k$,

$$
\begin{aligned}
\tilde{\mathcal{L}}\left(A_{1}^{*} \otimes\right. & \left.A_{2}^{*} \otimes \cdots \otimes A_{k}^{*}\right) \\
= & \sum_{B_{1}^{*}, B_{2}^{*}, \ldots, B_{\ell}^{*}} \sum_{a_{1}^{*}, a_{2}^{*}, \ldots, a_{k}^{*}} \sum_{b_{1}^{*}, b_{2}^{*}, \ldots, b_{\ell}^{*}} \mathcal{L}_{a_{1}^{*} \otimes a_{2}^{*} \otimes \cdots \otimes a_{k}^{*}, b_{1}^{*} \otimes b_{2}^{*} \otimes \cdots \otimes b_{\ell}^{*}} \\
& F_{g_{1}}^{\infty}\left(B_{1}, b_{1}\right) F_{g_{2}}^{\infty}\left(B_{2}, b_{2}\right) \ldots F_{g_{\ell}}^{\infty}\left(B_{\ell}, b_{\ell}\right) F_{f_{1}}^{\infty}\left(a_{1}, A_{1}\right) F_{f_{2}}^{\infty}\left(a_{2}, A_{2}\right) \\
& \ldots F_{f_{k}}^{\infty}\left(a_{k}, A_{k}\right) B_{1}^{*} \otimes B_{2}^{*} \otimes \cdots \otimes B_{\ell}^{*}
\end{aligned}
$$

where the first sum is over all $B_{1}^{*}, B_{2}^{*}, \ldots, B_{\ell}^{*}$ such that $B_{j}^{*}$ is a critical cosimplex of $g_{j}$, the second sum is over all $k$-tuples of cosimplices of $M$, and the third sum is over all $\ell$-tuples of cosimplices of $M$. Then

$$
\tilde{\mathcal{L}}: \mathcal{M}^{*}\left(f_{1}\right) \otimes \mathcal{M}^{*}\left(f_{2}\right) \otimes \cdots \otimes \mathcal{M}^{*}\left(f_{k}\right) \longrightarrow \mathcal{M}^{*}\left(g_{1}\right) \otimes \mathcal{M}^{*}\left(g_{2}\right) \otimes \cdots \otimes \mathcal{M}^{*}\left(g_{\ell}\right)
$$

induces $L$.

We can use Theorem 3.9 to construct more general Morse cochain maps which induce $L$. For each $i=1,2, \ldots, k$, let $h_{1}^{i}, h_{2}^{i}, \ldots, h_{p_{i}}^{i}$ denote a sequence of Morse functions and $r_{1}^{i}, r_{2}^{i}, \ldots, r_{p_{i}}^{i}$ a sequence of elements from $\mathbb{Z}_{\geq 0} \cup\{\infty\}$. For each $i=1,2, \ldots, k$, define a map $\Phi_{h^{i}}^{*}: C^{*}(M, \mathbb{Z}) \rightarrow C^{*}(M, \mathbb{Z})$, by

$$
\Phi_{h^{i}}^{*}=\left(\Phi_{h_{p_{i}}^{i}}\right)^{r_{p_{i}}} \ldots\left(\Phi_{h_{2}^{i}}\right)^{r_{2}}\left(\Phi_{h_{1}^{i}}\right)^{r_{1}} .
$$


Now define a map

$$
\Phi_{h}^{*}: \otimes^{k} C^{*}(M, \mathbb{Z}) \rightarrow C^{*}(M, \mathbb{Z})
$$

by

$$
\Phi_{h}^{*}=\Phi_{h^{1}}^{*} \otimes \Phi_{h^{2}}^{*} \otimes \cdots \otimes \Phi_{h^{k}} .
$$

Similarly, for each $i=1,2, \ldots, \ell$, let $\tilde{h}_{1}^{i}, \tilde{h}_{2}^{i}, \ldots, \tilde{h}_{q_{i}}^{i}$ denote a sequence of Morse functions and $\tilde{r}_{1}^{i}, \tilde{r}_{2}^{i}, \ldots, \tilde{r}_{q_{i}}^{i}$ a sequence of elements from $\mathbb{Z}_{\geq 0} \cup\{\infty\}$. For each $i=1,2, \ldots, \ell$, define a map $\Phi_{\tilde{h}^{i}}^{*}: C^{*}(M, \mathbb{Z}) \rightarrow C^{*}(M, \mathbb{Z})$, and $\Phi_{\tilde{h}}^{*}: \otimes^{\ell} C^{*}(M, \mathbb{Z}) \rightarrow$ $C^{*}(M, \mathbb{Z})$, in the analogous way.

Theorem 4.3. The map

$$
\begin{gathered}
\tilde{\mathcal{L}}=\pi_{\mathcal{M}} \circ\left(\Phi_{g}^{*}\right)^{\infty} \circ \Phi_{\tilde{h}}^{*} \circ \mathcal{L} \circ\left(\Phi_{f}^{*}\right)^{\infty} \circ \Phi_{h}^{*}: \mathcal{M}^{*}\left(f_{1}\right) \otimes \mathcal{M}^{*}\left(f_{2}\right) \otimes \cdots \otimes \mathcal{M}^{*}\left(f_{k}\right) \\
\longrightarrow \mathcal{M}^{*}\left(g_{1}\right) \otimes \mathcal{M}^{*}\left(g_{2}\right) \otimes \cdots \otimes \mathcal{M}^{*}\left(g_{\ell}\right)
\end{gathered}
$$

induces $L$.

\section{EXAMPLES}

Example 1. The Identity Map. Let

$$
I: H^{*}(M, \mathbb{Z}) \rightarrow H^{*}(M, \mathbb{Z})
$$

denote the identity map. This map is obviously induced by the identity map

$$
\mathcal{I}: C^{*}(M, \mathbb{Z}) \rightarrow C^{*}(M, \mathbb{Z}) .
$$

Let $f$ and $g$ be two Morse functions on $M$. Define the map

$$
\tilde{\mathcal{I}}: \mathcal{M}^{*}(f) \rightarrow \mathcal{M}^{*}(g)
$$

by setting, for every critical simplex $A$ of $f$,

$$
\tilde{\mathcal{I}}\left(A^{*}\right)=\sum_{B \in C r(g)} \tilde{\mathcal{I}}_{A, B} B^{*}
$$

where

$$
\tilde{\mathcal{I}}_{A, B}=\sum_{a} F_{g}^{\infty}(B, a) F_{f}^{\infty}(a, A),
$$

where the sum is over all simplices $a$ of $M$. Note that $F_{g}^{\infty}(B, a) F_{f}^{\infty}(a, A)=0$ unless $A, B$ and $a$ all have the same dimension. This shows that $\tilde{\mathcal{I}}$ maps $\mathcal{M}^{p}(f)$ to $\mathcal{M}^{p}(g)$. Then Corollary 4.2 implies the following theorem.

Theorem 5.1. The map $\tilde{\mathcal{I}}: \mathcal{M}^{*}(f) \rightarrow \mathcal{M}^{*}(g)$ induces the identity map on $H^{*}(M, \mathbb{Z})$.

This map $\tilde{\mathcal{I}}$ was described in the introduction and illustrated in Figure 0.2.

Note that if $B \neq a$, then $F_{g}^{\infty}(B, a)=0$ unless $V_{g}(a) \neq 0$, and this is true if and only if $a$ is in the image of $\left(V_{g}\right)^{*}$. Similarly, if $A \neq a$, then $F_{f}^{\infty}(a, A)=0$ unless $V_{f}^{*}(a) \neq 0$, and this is true if and only if $a$ is in the image of $V_{f}$.

An interesting special case is when $f=g$. Since the images of $V_{f}$ and $\left(V_{f}\right)^{*}$ are disjoint, $F_{f}^{\infty}(B, a) F_{f}^{\infty}(a, A)=0$ unless $a=A$ or $a=B$. In either case, $a$ is critical, so is in neither the image of $V_{f}$ nor $\left(V_{f}\right)^{*}$. Thus, we see that $F_{f}^{\infty}(B, a) F_{f}^{\infty}(a, A)=0$ unless $A=B=a$, in which case $F_{f}^{\infty}(B, A) F_{f}^{\infty}(a, A)=1$. This shows that if $f=g$, then the map $\tilde{\mathcal{I}}: \mathcal{M}^{*}(f) \rightarrow \mathcal{M}^{*}(g)$ is the identity map, just as one would expect. 
Theorem 4.3 can be used to construct more general Morse cochain maps which induce the identity map on cohomology. These maps are described in the introduction and illustrated in Figure 0.3.

Example 2. The Cup Product. Let

$$
\cup: H^{*}(M, \mathbb{Z}) \otimes H^{*}(M, \mathbb{Z}) \rightarrow H^{*}(M, \mathbb{Z})
$$

denote the cup product. There are many different cochain maps which induce the cup product on cohomology. To define one such map, which we also denote by $\cup$, choose a linear ordering of the vertices of $M$. Let $c^{*}$ be a $p$-cochain and $\tilde{c}^{*}$ a $q$-cochain. We will define a $(p+q)$-cochain $c^{*} \cup \tilde{c}^{*}$ as follows. Suppose that the vertices $x_{0}<x_{1}<\cdots<x_{p+q}$ span a simplex. Then we set

$$
c^{*} \cup \tilde{c}^{*}\left(\left[x_{0}, x_{1}, \ldots, x_{p+q}\right]\right)=c^{*}\left(\left[x_{0}, x_{1}, \ldots, x_{p}\right]\right) \tilde{c}^{*}\left(\left[x_{p}, x_{p+1}, \ldots, x_{p+q}\right]\right) .
$$

We now extend the map $\cup$ linearly to a map

$$
\cup: C^{*}(M, \mathbb{Z}) \otimes C^{*}(M, \mathbb{Z}) \rightarrow C^{*}(M, \mathbb{Z})
$$

and this map induces the map $\cup$ on cohomology.

Let $f_{1}, f_{2}$ and $g$ be Morse functions on $M$. We define a map

$$
\widetilde{U}: \mathcal{M}^{*}\left(f_{1}\right) \otimes \mathcal{M}^{*}\left(f_{2}\right) \rightarrow \mathcal{M}^{*}(g)
$$

as follows. For any critical cosimplices $A_{1}$ and $A_{2}$ of $f_{1}$ and $f_{2}$, resp., we set

$$
\widetilde{\cup}\left(A_{1}^{*} \otimes A_{2}^{*}\right)=\sum_{B} \sum_{a_{1} \cup a_{2}=b} F_{\infty}^{g}(B, b) F_{\infty}^{f_{1}}\left(a_{1}, A_{1}\right) F_{\infty}^{f_{2}}\left(a_{2}, A_{2}\right) B^{*} .
$$

Corollary 4.2 now implies the following theorem.

Theorem 5.2. The map $\widetilde{\cup}: \mathcal{M}^{*}\left(f_{1}\right) \otimes \mathcal{M}^{*}\left(f_{2}\right) \rightarrow \mathcal{M}^{*}(g)$ induces the cup product on cohomology.

This is the map described in the introduction and illustrated in Figure 0.4.

We observe that the maps $\cup$ and $\widetilde{\cup}$ are associative, but not commutative. We can define a cup product map on cochains with values in $\mathbb{Q}$ that is graded-commutative but not associative by averaging the cochain map $\cup$ over all orderings of the vertices of $M$. Applying Theorem 3.2 shows that if $f_{1}=f_{2}$, then the result of averaging $\widetilde{U}$ over all orderings of the vertices of $M$ yields a graded-commutative map on the rational Morse cochains which induces the cup product on cohomology.

Theorem 4.3 can be used to construct more general Morse cochain maps which induce the cup product on cohomology. These maps are described in the introduction and illustrated in Figure 0.5.

Example 3. The Euler Characteristic. Let

$$
I_{p}: H^{*}(M, \mathbb{R}) \rightarrow H^{*}(M, \mathbb{R})
$$

denote the identity map. Then the Euler characteristic is given by the alternating sum of the traces

$$
\chi(M)=\sum_{p=0}^{\operatorname{dim} M}(-1)^{p} \operatorname{trace}\left(I_{p}\right) .
$$

It is a standard fact in this subject that if

$$
V^{n} \stackrel{\delta}{\longleftarrow} V^{n-1} \stackrel{\delta}{\longleftarrow} V^{n-2} \stackrel{\delta}{\longleftarrow} \cdots
$$


is any cocomplex whose cohomology is isomorphic to $H^{*}(M, \mathbb{Z})$, and

$$
L_{P}: V^{p} \rightarrow V^{p}
$$

is any collection of maps which induce the identity map on cohomology, then the Euler characteristic is given by

$$
\chi(M)=\sum_{p=0}(-1)^{p} \operatorname{trace}\left(L_{p}\right) .
$$

Now let $f$ be a Morse function on $M$. We know from Example 1 how to construct maps from $\mathcal{M}^{*}(f)$ to itself which induce the identity map on cohomology. Namely, in the maps illustrated in Figures 0.2 and 0.3 we found maps from $\mathcal{M}^{*}(f)$ to $\mathcal{M}^{*}(g)$ which induce the identity map on cohomology; so now we simply set $f=g$. Moreover, since we will be taking the trace of this map, we need only consider sequences of gradient paths which go from an $f$-critical simplex to itself. The appropriate configuration of gradient paths is illustrated in Figure 0.6. An explicit formula is given in the following theorem.

Theorem 5.3. Let $f$ and $h_{1}, h_{2}, \ldots, h_{k}$ be Morse functions on $M$ for some $k \geq 0$, and, for $i=1,2, \ldots, k, r_{i} \in \mathbb{Z}_{\geq 0} \cup\{\infty\}$. Then

$$
\begin{gathered}
\chi(M)=\sum_{p=0}^{\operatorname{dim}(M)}(-1)^{p} \sum_{f \text {-critical } A^{(p)} \sum_{a_{0}, \ldots, a_{k}} F_{f}^{\infty}\left(A, a_{0}\right) F_{h_{1}}^{r_{1}}\left(a_{0}, a_{1}\right) F_{h_{2}}^{r_{2}}\left(a_{1}, a_{2}\right)} \ldots F_{h_{k}}^{r_{k}}\left(a_{k-1}, a_{k}\right) F_{f}^{\infty}\left(a_{k}, A\right) .
\end{gathered}
$$

Example 4. The Fundamental Class. Suppose that $M$ is a connected simplicial complex. Then one can consider the fundamental class $\alpha^{*} \in H^{0}(M, \mathbb{Z})$. This class assigns to every 0 -chain $\sum_{v} n_{v} v$, where the sum is over all vertices $v$ of $G$, the number $\sum_{v} n_{v}$. In order to place this discussion in the context of this paper, we consider the linear map

$$
\mathbb{C} \rightarrow H^{*}(M, \mathbb{Z})
$$

(where $\mathbb{C}$ denotes the complex numbers) which takes the number 1 to $\alpha^{*}$. The class $\alpha^{*}$ is represented by the cochain $c^{*}=\sum_{v} v^{*}$. Since $\mathbb{C}$ is the tensor product of 0 copies of $H^{*}(M, \mathbb{Z})$, this map fits into the discussion in the previous sections. Applying Corollary 4.2 to this simple example leads to the conclusion that for any Morse function $f, \alpha^{*}$ is represented by the cochain $\sum_{V} n_{V} V^{*}$, where the sum is over all critical vertices $V$, and

$$
n_{V}=\sum_{v} F^{\infty}(v, V)
$$

Equivalently, the cochain is constructed by counting all gradient paths as illustrated in Figure 0.7(i), with the endpoint being a critical vertex.

Suppose that $M$ is an $n$-dimensional pseudo-manifold. That is, every $(n-1)$ simplex is a face of exactly two $n$-simplices. If $M$ is orientable, then $H_{n}(M, \mathbb{Z}) \cong \mathbb{Z}$ and one can choose a generator $\alpha$. If we endow each $n$-simplex with the appropriate orientation, then the class $\alpha$ is represented by the $n$-chain $c=\sum_{a^{(n)}} a$. If $M$ is not orientable, then one can do the same working with coefficients in $\mathbb{Z}_{2}$. For any $n$ cochain $c^{*}=\sum_{\left(a^{n}\right)} c_{a^{*}} a^{*}, c^{*}(\alpha)=\sum_{a^{(n)}} c_{a^{*}}$. To place this discussion in the context 
of this paper, we should view the fundamental class as a map on cohomology. We can view the fundamental class $\alpha$ as a map

$$
\alpha: H^{*}(M, \mathbb{Z}) \rightarrow \mathbb{C}
$$

which maps a degree $n$ cohomology class to its evaluation on $\alpha$ and a class of degree $m$ to 0 if $m \neq n$. Let $f$ be a Morse function on $M$. Let us now construct a map $\tilde{\alpha}$ on Morse cochains that induces this map. If $m^{*}$ is a Morse $m$-cochain, and $m \neq n$, then certainly $\tilde{\alpha}\left(m^{*}\right)=0$ will do the job. If $m^{*}$ has degree $n$, then Corollary 4.2 implies that one should map a critical $n$-cosimplex $A^{*}$ to

$$
\sum_{a^{(n)}} F^{\infty}(a, A) a^{*}(c)=\sum_{a^{(n)}} F^{\infty}(a, A) .
$$

That is, we count all gradient paths from a general $n$-simplex to a critical $n$-simplex. However, there are no nontrivial gradient paths to a critical $n$-simplex. Thus, the only contribution to this sum is when $a=A$. That is, the map $\tilde{\alpha}$ maps each critical $n$-cosimplex (endowed with the appropriate orientation ) to 1 .

On the other hand, we may view the fundamental class $\alpha$ as a map

$$
\alpha: \mathbb{C} \rightarrow H_{*}(M, \mathbb{Z}),
$$

which takes 1 to $\alpha$. Although we only discussed operations on cohomology in the previous section, the same ideas can be used to construct maps on homology. In this case, the dual statement to Corollary 4.2 implies that the map $\alpha$ above is induced by the map $\mathbb{C} \rightarrow \mathcal{M}_{*}$ which takes 1 to $\sum_{A^{(n)}} \alpha_{A} A$ where the sum is over all critical $n$-simplices $A$, and

$$
\alpha_{A}=\sum_{a^{(n)}} F^{\infty}(A, a) .
$$

That is, we construct the fundamental class in Morse homology by counting all gradient paths from a critical $n$-simplex to an arbitrary $n$-simplex. This is precisely the map that is illustrated in Figure 0.7(ii).

\section{REFERENCES}

[1] E. Babson, A. Björner, S. Linussion, J. Shareshian and V. Welker, Complexes of not i-Connected Graphs, Topology, 38 (1999), pp. 271-299. MR 2000a:57001

[2] M. Betz and R. Cohen, Graph moduli space and cohomology operations, Turk. J. of Math., 18 (1994), pp. 23-41. MR 95i:58037

[3] M. Chari, On Discrete Morse Functions and Combinatorial Decompositions, Formal Power Series and Algebraic Combinatorics (Vienna, 1997), Discrete Math., 217 (2000), pp.101-113. MR 2001g:52016

[4] R. Forman, A Discrete Morse Theory for Cell Complexes, in Geometry, Topology and Physics for Raoul Bott, S.T. Yau (ed.), International Press, 1995. MR 97g:57030

[5] _ Morse Theory for Cell Complexes, Adv. in Math., 134 (1998), pp. 90-145. MR 99b:57050

[6] _ Witten-Morse Theory for Cell Complexes, Topology, 37 (1998), pp. 945-979. MR 99m:58046

[7] - Combinatorial Vector Fields and Dynamical Systems, Math. Zeit., 228 (1998), pp. 629-681. MR 99f:58165

[8] _ Combinatorial differential topology and geometry, in New Perspectives in Algebraic Combinatorics (Berkeley, CA. 1996-97), Math. Sci. Res. Inst. Publ. 38, Cambridge Univ. Press, Cambridge, 1999, pp. 177-206. MR 2000h:57041

[9] Morse Theory and Evasiveness, Combinatorica, 20 (2001), pp. 489-504. MR 2001k:57006

[10] , Novikov-Morse theory for cell complexes, to appear in Internat. J. of Math. 
[11] K. Fukaya, Morse homotopy, $A^{\infty}$-category, and Floer homologies, in Proc. Garc. Workshop on Geometry and Topology '93, (Seoul, 1993), Lecture Note ed. H. J. Kim, Lecture Note Ser. 18, Seoul National University, pp. 1-102. MR 95e:57053

[12] Morse homotopy and its quantization, in Geometric Topology (Athens, GA 1993), AMS/IP Stud. Adv. Math 21, Amer. Math. Soc., Providence RI, 1997, pp. 409440. MR 98i:57061

[13] J. Jonsson, On the homology of some complexes of graphs, preprint, 1998.

[14] The decision tree method, preprint, 1999.

[15] V. Mathai and S. G. Yates, Discrete Morse theory and extended $L^{2}$ homology, J. Funct. Anal. 168 (1999), 84-110. MR 2000i:58038

[16] J. Shareshian, Discrete Morse Theory for Complexes of 2-Connected Graphs, Topology, 40 (2001), pp. 681-701.

Department of Mathematics, Rice University, Houston, Texas 77251

E-mail address: forman@math.rice.edu

$U R L:$ http://math.rice.edu/ forman/ 